\title{
Desafíos y acciones de Terapia Ocupacional en salud mental. Tiempos de pandemia y transformación
}

\author{
Diana Milena Ramírez Osorio ${ }^{1}$ \\ Nidya Alejandra Jiménez Moreno² \\ Aida Navas ${ }^{3}$ \\ Margarita González ${ }^{4}$
}

Ramírez-Osorio, D.M., Jiménez-Moreno, N.A., Navas, A. y González, M. (2020). Desafíos y acciones de Terapia Ocupacional en salud mental. Tiempos de pandemia y transformación. Revista Ocupación Humana, 20(2), 3-9. https://doi.org/10.25214/25907816.1070

Aproximarnos a comprender la salud mental en la complejidad de una pandemia reafirma la necesidad de reconocerla como un conjunto de relaciones humanas y dinámicas sociales vinculadas a la garantía de los derechos fundamentales, al trabajo digno, a la justicia social, al acceso a servicios ciudadanos, al cuidado y el co-cuidado de la vida como práctica y visión personal y colectiva y, por supuesto, a las necesidades y sufrimientos subjetivos (Abadía-Barrero y Melo-Moreno, 2014).

Por esto, tal como lo menciona Granda (2004) en el marco de los planteamientos de la medicina social latinoamericana, es fundamental reflexionar y entender la salud y la vida desde la realidad de

\footnotetext{
1 Terapeuta ocupacional. Especialista en Gerencia de la Salud Pública. Máster en Políticas Sociales y Mediación Comunitaria. Secretaria del Consejo Directivo Nacional, Colegio Colombiano de Terapia Ocupacional. Docente Escuela Colombiana de Rehabilitación. Bogotá, Colombia. diana.ramirezo@ecr.edu.co iD https://orcid.org/0000$\underline{\text { 0001-8100-6305 }}$

${ }^{2}$ Terapeuta ocupacional. Magíster en Desarrollo Educativo y Social. Vicepresidenta del Consejo Directivo Nacional 2020, Colegio Colombiano de Terapia Ocupacional. Docente Universidad Nacional de Colombia. Bogotá, Colombia.najimenezmo@unal.edu.co iD https://orcid.org/0000-0003-2732-492

${ }^{3}$ Terapeuta ocupacional. Delegada del Colegio Colombiano de Terapia Ocupacional en el Consejo Nacional de Salud Mental. Bogotá, Colombia. aidanavas2001@yahoo.com iD https://orcid.org/0000-0003-0238-635x

${ }^{4}$ Terapeuta ocupacional. Máster en Ergonomía.Vicepresidenta Federación Mundial de Terapeutas Ocupacionales-WFOT. Bogotá, Colombia.magonza48@yahoo.es

iD https://orcid.org/0000-0002-8867-934X
} 
los sujetos, porque vivir y poder vivir dignamente genera salud. Hay que estimular el debate de la salud y la salud mental a partir de distintas perspectivas y actores para recrear y buscar alternativas que realmente apunten a la complejidad de las necesidades percibidas por las personas y las sociedades.

Es en este contexto que la Terapia Ocupacional ha venido aportando a la concepción de los procesos de salud-bienestar-enfermedad desde la perspectiva ocupacional y de la complejidad. La trayectoria histórica de la profesión en Colombia ha implicado importantes luchas de participación política, necesarias para hacer visibles sus saberes, competencias, quehaceres e impactos en temas de salud que tradicionalmente han estado inmersos en enfoques hegemónicos -biomédico, organicista, mecanicista y funcionalista-, los cuales, en nuestra práctica profesional se complementan con otras formas como la perspectiva biopsicosocial, la determinación social, la salud colectiva, la dialéctica o la complejidad social.

Respecto a los desafíos del manejo de la pandemia para el ejercicio profesional a escala global, la Federación Mundial de Terapeutas Ocupacionales (WFOT, por sus siglas en inglés) publicó una declaración de posicionamiento en la cual reconoce el impacto del COVID-19 en la vida, la salud y el bienestar de las personas, las comunidades y las familias (WFOT, 2020).

La Federación reconoce en esta declaración que el impacto en la ocupación y, en general, en la participación, el manejo de rutinas y el acceso a servicios y espacios comunitarios ha obligado a las personas a ajustar sus hábitos y a buscar formas de enfrentar los cambios, la incertidumbre, las nuevas formas de hacer, sentir, pensar e interactuar. Enfatiza igualmente en la importancia del trabajo de las y los terapeutas ocupacionales con grupos poblacionales de diferentes edades y condiciones, en escenarios en los cuales se busca dignificar la vida, así como promover la participación y la autonomía para el desarrollo de actividades del hogar, estudio, trabajo, ocio y todas aquellas que tienen sentido para las personas (WFOT, 2020).

La Federación hace un Ilamado a la sociedad en general a velar por el derecho fundamental que tienen todas las personas a recibir atención integral y rehabilitación, a que se promueva la salud y se garantice la inclusión social y ocupacional de las personas afectadas por el COVID-19. Esto implica fortalecer el liderazgo social, político, académico, científico y gremial, basándose en el compromiso del colectivo de terapeutas ocupacionales para la generación de investigaciones y estrategias de abordaje que respondan a las complejas dinámicas actuales (WFOT, 2020).

Por otra parte, los resultados de la encuesta realizada por la WFOT el año anterior, en la cual participaron 2.750 terapeutas ocupacionales de 100 países, resaltan la importancia de la intervención durante y después de la pandemia de COVID-19 para producir nuevos lineamientos, aprendizajes y oportunidades de información para la comunidad internacional a partir de investigaciones y estudios basados en la evidencia (WFOT, 2021). 
En este contexto, el manejo de la pandemia de COVID-19 nos interpela como profesionales a comprender los impactos del aislamiento físico, las restricciones asociadas al riesgo de enfermar y el surgimiento de nuevas formas de interacción social -principalmente mediadas por la virtualidad-sobre nuestra relación con los espacios y los tiempos en los que solíamos desarrollar nuestras vidas.

A su vez, las condiciones de empleo y trabajo en todo el mundo se han visto afectadas, lo que puede generar situaciones de deprivación y apartheid ocupacional (Kronenberg et al., 2006); en entornos como el colombiano, esto profundiza las desigualdades e injusticias sociales. Entre tanto, las percepciones de inseguridad, miedo y paranoia han impactado negativamente el tejido social, lo que sumado a las situaciones antes mencionadas señala un impacto relevante en la salud física y mental.

En este sentido es preciso asumir el desafío de liderar procesos que permitan dar continuidad a los cuidados y a la participación en escenarios cotidianos como el hogar, la escuela, el trabajo y la comunidad; así mismo, estructurar alternativas de abordaje que respondan a las nuevas demandas de interacción mediadas por la virtualidad y por los protocolos de bioseguridad, teniendo en cuenta que las interacciones presenciales están fuertemente arraigadas en nuestra sociedad y hacen parte de nuestra cultura. Igualmente, fortalecer acciones de formulación y puesta en marcha de políticas y atención directa a personas y familias afectadas por el COVID-19; seguimiento y continuidad de los cuidados en contextos cotidianos; atención de emergencias psicosociales, y abordaje psicosocial de situaciones que afecten la salud mental de la población.

En el escenario actual, la Terapia Ocupacional centrada en las personas y basada en la ocupación (Trujillo et al., 1995; Kielhofner, 2006; Grupo de Investigación Ocupación y Realización Humana, 2011; Galheigo, 2019) constituye una propuesta potente para la construcción de sentidos y significados como factor socializador; como factor estructural en las nociones de tiempo y espacio, y como factor protector. Esta visión es necesaria para reconocernos como sociedad y reconstruir o resignificar vínculos entre personas, colectivos, espacios e instituciones.

El Colegio Colombiano de Terapia Ocupacional, en cumplimiento de su objetivo misional de incidir en políticas públicas y con base en las disposiciones de la Ley 1616/2013, ha participado desde el año 2015 en el Consejo Nacional de Salud Mental. En este espacio de análisis y asesoría ha apoyado la construcción de documentos oficiales como la Política Nacional de Salud Mental (Resolución 4886/2018), la Política Integral para la Prevención y Atención al Consumo de Sustancias Psicoactivas (Resolución 89/2019) y el Documento CONPES (Consejo Nacional de Política Económica y Social) 3992 de 2020: Estrategia para la Promoción de la Salud Mental en Colombia, entre otros. 
La participación de diferentes sectores y actores sociales en el Consejo Nacional de Salud Mental muestra el valor del trabajo mancomunado y solidario. En este espacio se han dinamizado y afirmado reflexiones, experiencias, saberes y evidencias sobre el cuidado de la salud mental y la atención de los trastornos y las enfermedades mentales. Una vez se declaró la pandemia, se activaron de inmediato estrategias de respuesta. El Colegio Colombiano de Terapia Ocupacional y el Consejo Nacional de Trabajo Social pusieron en marcha una propuesta que implicó la construcción conjunta de alternativas, en las cuales se entendió que la atención a la población en la pandemia debía verse de manera integral e integrada, no solo desde el abordaje de la crisis, sino también desde la perspectiva de la ética y la continuidad de los cuidados. Entendimos que la cultura del autocuidado, del cuidado del otro y del cuidado del entorno en el que tiene lugar la vida cotidiana permitirá mitigar los impactos que sobre la salud mental de la población puedan generar el distanciamiento físico y las demás variables antes mencionadas.

En este sentido, se identificó que la reconstrucción de las redes de apoyo y el fortalecimiento del tejido social y del entramado de sentidos y significados que las personas construyen en sus vidas a partir de su quehacer diario debían ser aspectos prioritarios en las acciones de apoyo que se implementaran. Con base en las iniciativas ejecutadas previamente por profesionales de Psiquiatría y Psicología, se propuso diseñar e implementar estrategias que permitieran a trabajadoras y trabajadores sociales y a terapeutas ocupacionales apoyar la promoción de la salud mental, la convivencia, la participación social y el equilibrio ocupacional de personas y comunidades.

Para estructurar la propuesta, el Consejo Nacional de Trabajo Social y el Colegio Colombiano de Terapia Ocupacional articularon los saberes y prácticas de ambas profesiones en estrategias interdisciplinares para el abordaje desde la perspectiva comunitaria y de apoyo a la gestión de las situaciones de vulnerabilidad evidenciadas en las personas y los colectivos por efectos de la pandemia. En la ejecución de la propuesta participó un número importante de terapeutas ocupacionales que atendieron a la convocatoria del Colegio y se integraron al equipo de trabajo interdisciplinar. Posteriormente se concertaron las temáticas y las metodologías para la puesta en marcha de las estrategias, con el apoyo logístico y de los medios de comunicación oficiales del Ministerio de Salud y Protección Social.

La propuesta integró cuatro líneas de trabajo:

1. Apoyo a las líneas de atención en crisis dispuestas por el Ministerio de Salud y Protección Social. Incluyeron atención directa, seguimiento a situaciones canalizadas, acciones colectivas de diálogo abierto, estrategias de psicoeducación y acciones indirectas;

2. Formación y asesoría al talento humano de las dos profesiones;

3. Abordaje interdisciplinar a territorios;

4. Investigación. 
La propuesta se replicó en dos acciones psicoeducativas de apoyo a profesionales del Instituto Colombiano de Bienestar Familiar (ICBF) que lideran procesos de atención directa a la población en la pandemia. Esta gestión se realizó conjuntamente con el Departamento de Pediatría de la Universidad Nacional de Colombia.

Fue así como se concretaron seis espacios de psicoeducación en los cuales se abordaron temáticas relacionadas con los haceres y las cotidianidades; el manejo de situaciones nuevas y la incertidumbre; el cuidado de la salud mental; el envejecimiento; el trabajo y el manejo de la carga laboral. Estos espacios animaron la interacción y la participación de quienes asistieron desde la postura crítica y reflexiva que amerita este momento.

En el desarrollo de esta propuesta, las terapeutas ocupacionales participantes reafirmaron la importancia de reconocer las diversidades y subjetividades como formas de resistencia a la normalización y la homogeneización. A su vez se dio paso, a partir de bases humanistas y sociales, a la comprensión de realidades personales y locales relacionadas con la salud y el bienestar, y a la construcción de saberes y prácticas necesarias para comprender y abordar los retos y las complejidades que demandan las formas de hacer y de ocuparse.

El proceso de diálogo y construcción interdisciplinar nos enseñó sobre lo importante del aprendizaje con otros y otras, nos convocó a la creatividad y a la innovación en las propuestas para la comunidad y en el uso de distintos recursos, dispositivos y habilidades. Nos sensibilizó sobre las oportunidades de esperanzar, apoyar y acompañar en un momento en el que todos y todas experimentamos incertidumbre, cambio y caos; es decir, nos atravesó la salud mental y la necesidad de cultivarla. Ya no hablábamos de otras personas a las que les pasaba o necesitaban algo, sino que nuestra propia experiencia de vida fue un mecanismo de captura y de proposición. Nos permitió comprender y animar la participación activa de la ciudadanía en la construcción y la vivencia de las políticas sociales.

En ese mismo sentido, la experiencia y todo el proceso de gestión también nos impulsa a seguir consolidando y a movilizar un pensamiento sistémico y complejo en el que la diversidad de saberes, la complementariedad, el diálogo abierto y la construcción de comunidades profesionales, académicas y civiles son potentes y necesarios para responder a las demandas de los entornos local, regional y global.

La permanente retroalimentación obtenida de las personas participantes y su interés por apropiarse del espacio dispuesto como oportunidad para trabajar en una co-construcción propia, con sus familias y comunidades, nos llevan a vislumbrar perspectivas de cohesión social que transformen la salud mental. Unido a lo anterior, los análisis realizados al finalizar el año 2020 con profesionales del Ministerio de Salud y Protección Social, positivos y motivadores, nos hacen pensar en la importancia de darle continuidad a la ejecución de la propuesta. 
Adicionalmente, la experiencia vivida ha sido útil para apreciar la virtualidad como una herramienta que puede contribuir a cerrar la brecha entre las necesidades de atención en salud mental, el sufrimiento y la vulnerabilidad de la población colombiana, y las estrategias de abordaje disponibles. Su instauración como recurso para la interacción social permitió encuentros plurales entre líderes, profesionales y personas de la sociedad civil de diferentes territorios y contextos, a través de los cuales se reconoció la necesidad de que la salud mental esté en la agenda pública y ocupe un lugar prioritario en la vida y la dinámica social.

Por último, nos deja inquietudes sobre lo que debemos cultivar como sociedad para enfrentar los cambios y las crisis. ¿De dónde sacar recursos afectivos cuando el tejido social ha sido históricamente lesionado y hay poca credibilidad en las posibilidades de una transformación efectiva y justa? ¿Cómo reconstruir el tejido social y la colectividad a partir de nuestras formas de hacer en la cotidianidad? ¿Cómo asumimos desde la Terapia Ocupacional el compromiso político de documentar y sistematizar nuestras prácticas locales para hacerlas visibles en el contexto global? 


\section{Referencias}

Abadía-Barrero, C. y Melo-Moreno, M. (2014). Repensar la salud desde una academia crítica y comprometida. Vida, acumulación y emancipación. Revista Gerencia y Políticas de Salud, 13(27), 41-57. http://dx.doi. org/10.11144/Javeriana.rgyps13-27.rsda

Congreso de la República de Colombia (2013, 21 de enero). Ley 1616 de 2013. Por medio de la cual se expide la ley de Salud Mental y se dictan otras disposiciones. Diario Oficial 48.680. www.secretariasenado.gov.co/senado/basedoc/ ley 1616 2013.html

Consejo Nacional de Política Económica y Social [CONPES] (2020, 14 de abril). Documento CONPES 3992. Estrategia para la promoción de la salud mental en Colombia. https://colaboracion.dnp.gov.co/CDT/ Conpes/Econ\%C3\%B3micos/3992.pdf

Galheigo, S. (2019, 23 de septiembre). Terapia Ocupacional crítica y la construcción de prácticas emancipadoras [Conferencia]. X Congreso Argentino y XII Congreso Latinoamericano de Terapia Ocupacional, Construyendo justicia ocupacional desde la perspectiva latinoamericana, Tucumán, Argentina.

Granda, E. (2004). ¿A qué Ilamamos salud colectiva hoy? Revista Cubana de Salud Pública, 30(2), 1-20. http://scielo. sld.cu/scielo.php?script=sci arttext\&pi$\mathrm{d}=$ S0864-34662004000200009

Grupo de Investigación Ocupación y Realización Humana (2011). Ocupación: sentido, realización y libertad. Diálogos ocupacionales en torno al sujeto, la sociedad y el medio ambiente. Universidad Nacional de Colombia.
Kronenberg, F., Simó-Algado, S. y Pollard, N. (2006). Terapia Ocupacional sin fronteras. Aprendiendo del espiritu de los supervivientes. Médica Panamericana.

Kielhofner, G. (2006). Fundamentos conceptuales de terapia ocupacional. Médica Panamericana.

Ministerio de Salud y Protección Social (2018, 7 de noviembre). Resolución 4886 de 2018. Por la cual se adopta la Política Nacional de Salud Mental. Diario Oficial 46.709. https://www.minsalud.gov.co/ Normatividad Nuevo/Forms/DispForm. aspx? ID =5385

Ministerio de Salud y Protección Social (2019, 16 de enero). Resolución 89 de 2019. Por la cual se adopta la Política Integral para la Prevención y Atención del Consumo de Sustancias Psicoactivas. Diario Oficial 50858. https://www.minsalud.gov.co/ sites/rid/Lists/BibliotecaDigital/RIDE/DE/ Dl]/resolucion-089-de-2019.pdf

Trujillo, A. (1995). Desempeño ocupacional realizante: un modelo sobre los propósitos de la terapia ocupacional. Revista Ocupación Humana, 6(2), 11- 21.

World Federation of Occupational Therapists [WFOT] (2020). WFOT's public statement on occupational therapy and rehabilitation of people affected by the COVID-19 pandemic. WFOT. https://wfot.org/ resources/occupational-therapy-and-rehabilitation-of-people-affected-by-the-covid-19-pandemic

World Federation of Occupational Therapists [WFOT] (2021). Global survey report - the impact of covid-19 for occupational therapy. WFOT. https://www.wfot.org/resources/global-survey-report-the-impact-of-covid-19-for-occupational-therapy 